\title{
Gauge Invariances and Phases of Massive Higher Spins in (Anti-) de Sitter Space
}

\author{
S. Deser* and A. Waldron ${ }^{\dagger}$ \\ Physics Department, Brandeis University, Waltham, Massachusetts 02454
}

(Received 23 February 2001; published 27 June 2001)

\begin{abstract}
The $\left(m^{2}, \Lambda\right)$ plane of spin $s>1$ massive fields in (anti-) de Sitter space backgrounds is shown to consist of separate phases, divided by lines of novel "partially massless" gauge theories that successively remove helicities, starting from the lowest, 0 or $\pm 1 / 2$. The norms of the excluded states flip as the gauge lines are crossed and only the region containing the massive Minkowski theory is unitary. The partially massless gauge theories are unitary or not, depending on the ordering of the gauge lines. This "level splitting" of massless Minkowski gauge theories is specific to nonzero $\Lambda$.
\end{abstract}

DOI: $10.1103 /$ PhysRevLett.87.031601

The search for a Higgs as generator of mass is a central endeavor of modern particle physics. At the same time there is mounting evidence that our universe has a nonvanishing cosmological constant [1]. It is therefore extremely important to understand what mass means in cosmological backgrounds, also as string theory in cosmological backgrounds has led to significant theoretical advances. We find that conventional flat space notions must be extended for spins $s>1$. Although no fundamental $s>1$ particles have been observed and there is a large disparity between cosmological and nuclear scales, our results are relevant to all these research areas.

Gauge invariance, masslessness, null propagation, and strictly helicity $\pm s$ excitations are all synonymous in flat space, a degeneracy that is lifted in (anti-) de Sitter [(A)dS]. We show that, for massive spin $s>1$ theories, new gauge invariances allow "partially massless" propagation. Specifically, there are intermediate theories with $2,4, \ldots, 2 s, 2 s+1$ propagating degrees of freedom (PDOF) for bosons or $2,4, \ldots, 2 s$ PDOF for fermions. [The term helicity is used in a correspondence sense with Minkowski space. Also, a new feature for $s \geq 5 / 2$ is that auxiliary fields are necessary: in the massless limit they propagate but decouple. In passing, we note that conformal null propagation is generically also lost in (A)dS [2].]

The physics of massive higher spin fields in (A)dS is best displayed in the $\left(m^{2}, \Lambda\right)$ plane where, phases describing $2 s+1$ massive PDOF are separated by lines where gauge invariances remove subsets of lowest helicity modes. Further, the norms of the excised helicities flip sign as these gauge lines are crossed. As a consequence, (i) only the phase occupied by the flat massive theory is unitary, since the set of excitations flipping sign is distinct on each line. (ii) Unitarity of the partially massless theories requires that their gauge invariances remove any negative norm states. This occurs only when, starting from the unitary Minkowski region, the line removing the lowest helicity state(s) can be traversed first. Subsequent lines must also be traversed in order, ending on the strictly massless helicity $\pm s$ line.
PACS numbers: 11.10.Kk, 03.65.Pm, 04.62.+v, 11.15.-q

To see how massive constraints are transmuted to Bianchi identities along lines in the $\left(\mathrm{m}^{2}, \Lambda\right)$ plane, consider first the $s=1$ Proca equation $G_{\mu} \equiv\left(D^{2}-\right.$ $\left.m^{2}-\Lambda\right) \phi_{\mu}-D_{\mu} D \cdot \phi=0$ whose divergence $D \cdot G=$ $-m^{2} D \cdot \phi$ implies $3=2 s+1$ PDOF. Along the line $m^{2}=0$, the constraint becomes a Bianchi identity reflecting the gauge invariance $\delta \phi_{\mu}=D_{\mu} \xi$ and the system describes helicities \pm 1 only. For $s>1$, however, two new things happen: (i) The field equations have $[s]$ open indices, so each of $[s]$ successive divergences yields constraints implying $2 s+1$ PDOF. (ii) The monomial $\mathrm{m}^{2}$ in the Proca constraint is generalized to nontrivial functions of $(m, \Lambda)$ whose vanishing yields new Bianchi identities, an effect that clearly degenerates at $\Lambda=0$.

Each Bianchi identity implies gauge invariances which remove corresponding lower helicities from the spectrum. In addition, the coefficients of the divergence constraints flip sign across the gauge boundaries and appear as denominators in canonical (anti)commutators. Hence, whenever gauge invariance removes a particular helicity, its norm has opposite signs in the adjacent massive phases and the $\left(m^{2}, \Lambda\right)$ plane is partitioned into unitarily allowed and forbidden regions. [The constant curvature background does not suffer from the usual gravitational coupling problems in the unitarily allowed regions, although the old causality difficulties of $s=3 / 2$ in electromagnetic (e.m.) backgrounds [3,4] resurface for the forbidden regions.]

In this Letter, we explicitly treat spins $s=3 / 2,2,5 / 2$, and 3. It is known that, beyond its vector gauge invariance at $m^{2}=0$, spin 2 is partially massless along the line $m^{2}=2 \Lambda / 3$ thanks to a Weyl-like scalar gauge invariance and accompanying double divergence Bianchi identity [2]. The dS region $m^{2}<2 \Lambda / 3$ is unitarily forbidden [5]. We extend this result to spin $3 / 2$ and then show that spins $5 / 2$ and 3 possess new partially massless gauge lines. Finally we present a PDOF counting argument indicating that partial masslessness is enjoyed by all spins $s>3 / 2$ in $(\mathrm{A}) \mathrm{dS}$.

The massive spin $3 / 2$ field equation is (all our field equations are derived from Hermitian actions) 


$$
\begin{gathered}
\mathcal{R}_{\mu} \equiv \gamma_{\mu \nu \rho} \mathcal{D}^{\nu} \psi^{\rho}=0, \quad \mathcal{D}_{\mu} \equiv D_{\mu}+\frac{1}{2} m \gamma_{\mu}, \\
{\left[D_{\mu}, D_{\nu}\right] \psi_{\rho}=\frac{2 \Lambda}{3} g_{\rho[\mu} \psi_{\nu]}+\frac{\Lambda}{6} \gamma_{\mu \nu} \psi_{\rho} .}
\end{gathered}
$$

To test for Bianchi identities, compute the "curly" divergence of (2)

$$
\mathcal{D} \cdot \mathcal{R}=-\frac{3}{2}\left(m^{2}+\Lambda / 3\right) \gamma \cdot \psi .
$$

This is obviously a constraint whose coefficient vanishes along the gauge boundary $m^{2}=-\Lambda / 3$ familiar from cosmological supergravity [6]. Along this line the theory is gauge invariant under variations $\delta \psi_{\mu}=\mathcal{D}_{\mu} \varepsilon$ and describes only helicities $\pm 3 / 2$ in $\operatorname{AdS}(\Lambda<0)$.

The appearance of a ghost across this line can be detected by examining the 0 components of the equal time anticommutator (this method was originally used to exhibit the inconsistency of spin 3/2 e.m. couplings [3])

$$
\begin{aligned}
\left\{\psi_{0}(t, \vec{x}), \psi_{0}^{\dagger}\left(t, \vec{x}^{\prime}\right)\right\}= & \frac{\cosh ^{-2}(M t)\left(-{ }^{(3)} D^{2}-\Lambda / 4\right)}{3\left(m^{2}+\Lambda / 3\right)} \\
& \times \frac{1}{\sqrt{-g}} \delta^{3}\left(\vec{x}-\vec{x}^{\prime}\right)
\end{aligned}
$$

in the synchronous metric $d s^{2}=-d t^{2}+\cosh ^{2}(M t) \times$ $\left[d r^{2}+\sin ^{2}(M r)\left(d \theta^{2}+\sin ^{2} \theta d \phi^{2}\right)\right], M^{2} \equiv \Lambda / 3$, that describes dS, and also AdS when continued to imaginary $M$. Unitarity requires positivity of the operator on the right-hand side of (4). For AdS (ignoring spatial boundary terms) the intrinsic 3-Laplacian $-{ }^{(3)} D^{2}$ and $-\Lambda / 4$ are separately positive. In $\mathrm{dS}$, the lowest eigenvalue of ${ }^{(3)} D^{2}$ acting on spinors is $\Lambda / 4$ (the spatial slices are an $S^{3}$ there) so ${ }^{(3)} D^{2}-\Lambda / 4 \geq 0$. Therefore, the coefficient $m^{2}+\Lambda / 3$ in the would-be Bianchi identity (3) controls the sign of the anticommutator (4) and only the region $m^{2} \geq-\Lambda / 3$ is unitary. [As a consequence the $m \rightarrow 0$ limit can be taken only in dS, contrary to [7]. As discussed in [8], the true massless limit is the AdS one, $m \rightarrow \sqrt{-\Lambda / 3}$. This tuning of $(m, \Lambda)$ also implies that unitary supersymmetric representations exist only in AdS [9]. Unitary nonsupersymmetric models in dS are, of course, not ruled out.] The phase diagram follows.

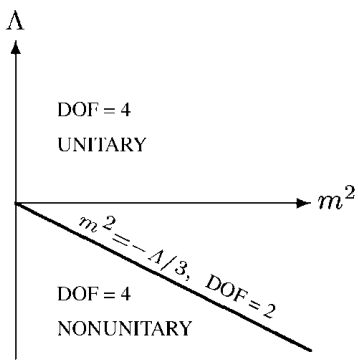

We emphasize that such poles in canonical (anti)commutators at gauge values of $\left(m^{2}, \Lambda\right)$ are a general feature of massive higher spins.

Spin 2 has two gauge lines as shown in the following phase diagram.

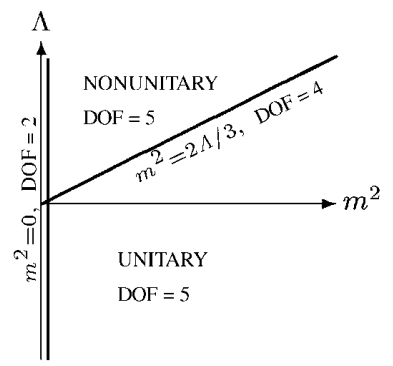

Linearized cosmological Einstein gravity remains at $m^{2}=$ 0 , so the $\left(\mathrm{m}^{2}, \Lambda\right)$ half plane is divided into two distinct regions, just as for spin $3 / 2$. The new partially massless gauge line at $m^{2}=2 \Lambda / 3$ is the Weyl-like, null, theory of [2]. A detailed canonical analysis proves that it describes 4 unitary PDOF [10] corresponding to the upper helicities $( \pm 2, \pm 1)$. The dS region $m^{2}<2 \Lambda / 3$ is unitarily forbidden [5] by an analysis equivalent to that of spin $3 / 2$ above.

The spin $5 / 2$ field equations are

$$
\begin{aligned}
\mathcal{R}_{\mu \nu}= & \not D \psi_{\mu \nu}+g_{\mu \nu}\left(\gamma \cdot D \cdot \psi-\frac{1}{2} \not D \psi_{\rho}{ }^{\rho}\right)+\gamma_{(\mu}\left(D_{\nu)} \psi_{\rho}{ }^{\rho}+2 \not D \gamma \cdot \psi_{\nu)}-2 D \cdot \psi_{\nu)}\right)-2 D_{(\mu} \gamma \cdot \psi_{\nu)} \\
& +m\left(\psi_{\mu \nu}-2 \gamma_{(\mu} \gamma \cdot \psi_{\nu)}-\frac{1}{2} g_{\mu \nu} \psi_{\rho}{ }^{\rho}\right)-\frac{5}{12} \mu g_{\mu \nu} \chi, \quad \mathcal{R}_{5}=-\alpha(\not D-3 m) \chi-\frac{5}{12} \mu \psi_{\rho}{ }^{\rho},
\end{aligned}
$$

where the spinor $\chi$ (with its equation of motion $\mathcal{R}_{5}$ ) exemplifies the auxiliary fields needed for $s \geq 5 / 2$ to impose $2 s+1$ massive PDOF. (See [11] for Minkowski higher spin massive field equations. They can also be derived by the Kaluza-Klein reduction [12] from their simpler $d=5$ massless antecedents [13] and the constraints then follow from the $d=5$ Bianchi identities [10].) Its coupling $\mu$ is uniquely fixed, $\mu^{2}=\frac{12 \alpha}{5}\left(m^{2}+4 \Lambda / 3\right)$, and it decouples on the strictly massless line $m^{2}=-4 \Lambda / 3$. The parameter $\alpha$ is free, modulo requiring reality of $\mu$ (and of the action). It can be set to 1 when $m^{2}>-4 \Lambda / 3$ and -1 for $m^{2}<-4 \Lambda / 3$. This discontinuity is a precursor of the nonunitary behavior we find for the massive and partially massless theories in the latter region.

Remarkably, the field equations enjoy two distinct gauge invariances,

$$
\begin{gathered}
\delta \psi_{\mu \nu}=D_{(\mu} D_{\tilde{\nu})} \varepsilon+\frac{5 \Lambda}{16} g_{\mu \nu} \varepsilon, \\
\delta \chi=-\frac{\sqrt{15 \alpha \Lambda}}{8 \alpha}(\not D+\sqrt{-3 \Lambda}) \varepsilon, \\
\delta \psi_{\mu \nu}=D_{(\mu} \varepsilon_{\tilde{\nu})}+\frac{1}{2} \sqrt{\frac{-\Lambda}{3}} \gamma_{(\mu} \varepsilon_{\tilde{\nu})}, \quad \delta \chi=0,
\end{gathered}
$$


along the respective AdS gauge lines

$$
m^{2}+\Lambda / 3=0, \quad m^{2}+4 \Lambda / 3=0 .
$$

[A tilded index denotes its gamma-traceless part, $X_{\tilde{\mu}} \equiv$ $X_{\mu}-(1 / 4) \gamma_{\mu} \gamma \cdot X$. We denote the symmetric-traceless part of a pair of indices by $X_{\{\mu \nu\}} \equiv X_{(\mu \nu)}-$ $(1 / 4) g_{\mu \nu} X_{\rho}{ }^{\rho}$ and symmetrize with unit weight.] The vector-spinor invariance yields the strictly massless theory, with helicities $\pm 5 / 2$, whereas the novel spinor invariance (6) removes helicities $\pm 1 / 2$, leaving $( \pm 5 / 2, \pm 3 / 2)$. Each invariance is accompanied by a Bianchi identity, which turns into a constraint ensuring $6=2 s+1$ massive PDOF when $\left(m^{2}, \Lambda\right)$ are detuned from their gauge values (8).

The combinations $\left(m^{2}+4 \Lambda / 3\right)$ and $\left(m^{2}+\Lambda / 3\right)$ control the positivity of equal time anticommutators. Therefore, since the gauge lines all lie in AdS, the $\left(m^{2}, \Lambda\right)$ plane is divided into three regions, only the one including Minkowski space $m^{2}>-4 \Lambda / 3$ being unitary as shown in the diagram.

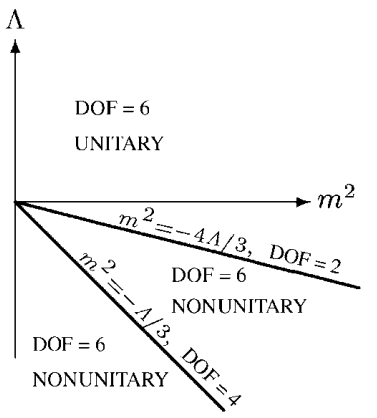

Although the strictly massless AdS $m^{2}=-4 \Lambda / 3$ theory is unitary, the partially massless one is not, as it fails the line ordering requirement: Starting from the unitary Minkowski region where all norms are positive, one would like first to traverse the line $m^{2}=-\Lambda / 3$, but that is possible only in dS with negative $m^{2}$ (imaginary values of $m$ violate Hermiticity of the action and unitary evolution). Crossing the AdS strictly massless line $m^{2}=-4 \Lambda / 3$ first flips the norm of helicities $( \pm 3 / 2, \pm 1 / 2)$ so the partially massless AdS theory cannot be unitary.

Spin 3 enjoys new unitary partially massless gauge lines as shown.

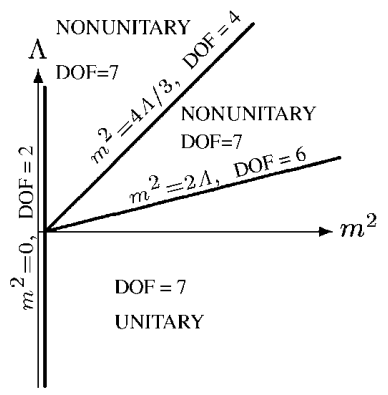

The field equations are

$$
\begin{aligned}
G_{\mu \nu \rho}= & \left(D^{2}-m^{2}+\Lambda / 3\right) \phi_{\mu \nu \rho}-3 D_{(\mu} D \cdot \phi_{\nu \rho)} \\
& +3 D_{(\mu} D_{\nu} \phi_{\rho) \sigma}{ }^{\sigma} \\
& -3 g_{(\mu \nu}\left[\left(D^{2}-m^{2}+2 \Lambda\right) \phi_{\rho) \sigma}{ }^{\sigma}\right. \\
& \left.\quad D \cdot D \cdot \phi_{\rho) \sigma^{\sigma}}{ }^{(9)} \frac{1}{2} D_{\rho)} D \cdot \phi_{\sigma}{ }^{\sigma}\right] \\
& +m g_{(\mu \nu} D_{\rho) \chi}=0, \\
G_{5}= & -2\left(D^{2}-4 m^{2}+8 \Lambda\right) \chi-m D \cdot \phi_{\sigma}{ }^{\sigma}=0,
\end{aligned}
$$

and the auxiliary $\chi$ decouples in the strictly massless limit $m=0$.

Here we find three new gauge invariances

$$
\begin{gathered}
\delta \phi_{\mu \nu \rho}=D_{(\mu} D_{\{\nu} D_{\rho\})} \xi+\frac{\Lambda}{2} g_{(\mu \nu} D_{\rho)} \xi, \\
\delta \chi=-\frac{1}{2} \sqrt{\frac{\Lambda}{2}}\left(D^{2}+\frac{10 \Lambda}{3}\right) \xi \\
\delta \phi_{\mu \nu \rho}=D_{(\mu} D_{\{\nu} \xi_{\rho\})}+\frac{\Lambda}{3} g_{(\mu \nu} \xi_{\rho)}, \\
\delta \chi=-\frac{1}{2} \sqrt{\frac{\Lambda}{3}} D \cdot \xi \\
\delta \phi_{\mu \nu \rho}=D_{(\mu} \xi_{\{\nu \rho\})}, \quad \delta \chi=0,
\end{gathered}
$$

along lines

$$
m^{2}-2 \Lambda=0, \quad m^{2}-4 \Lambda / 3=0, \quad m^{2}=0,
$$

respectively. Again the accompanying Bianchi identities become the massive constraints ensuring $7=2 s+1$ PDOF for detuned $\left(m^{2}, \Lambda\right)$.

The factors $m^{2}-2 \Lambda, m^{2}-4 \Lambda / 3$, and $m^{2}=0$ control positivity of lower helicity equal time commutators and therefore unitarity: Starting from the unitary Minkowski region $m^{2}>2 \Lambda$ we first encounter the gauge line $m^{2}=$ $2 \Lambda$. This is a unitary partially massless dS theory of helicities $( \pm 3, \pm 2, \pm 1)$ since the invariance (10) excises the lowest helicity 0 state, which reemerges as a ghost in the nonunitary region $4 \Lambda / 3^{2}<2 \Lambda$. At the next gauge line $m^{2}=4 \Lambda / 3$, the vector invariance (11) removes the dangerous helicities $(0, \pm 1)$ whose commutators both flip sign upon entering the final forbidden region $m^{2}<4 \Lambda / 3$ where helicities \pm 1 are now ghostlike. The boundary at $m^{2}=0$ is the strictly massless unitary theory where only helicities \pm 3 remain thanks to the traceless-symmetric invariance (12).

Simple counting arguments imply that novel gauge invariances and partially massless theories cutting the $\left(m^{2}, \Lambda\right)$ plane into unitarily allowed and forbidden regions are generic: Define the number of components $(\underline{s})$ of a symmetric $s$-index tensor $\phi_{\mu_{1} \ldots \mu_{s}}$ as $(\underline{s}) \equiv(s+1)(s+2)(s+3) / 3$ ! $[(\underline{s}) \equiv 0$ for $s<0]$ as well as $(\underline{s})^{\mathrm{T}}=(\underline{s})-(\underline{s-2})\left[=(s+1)^{2}\right.$ for $\left.s \geq 0\right]$ when requiring tracelessness and $(\underline{s})^{\mathrm{TT}}=(\underline{s})-(\underline{s-4})$ $\left[=2\left(s^{2}+1\right)\right.$ for $\left.s \geq 1\right]$ for double tracelessness. 
Consider first massless bosons. The field content is a double-traceless $s$ index symmetric tensor and the $s-1$ index gauge parameter is traceless:

\begin{tabular}{c|c} 
Fields & $(\underline{s})^{\mathrm{TT}}$ \\
\hline - Gauge & $2(\underline{s-1})^{\mathrm{T}}$
\end{tabular}

i.e., $2(\underline{s-1})^{\mathrm{T}}$ DOF can be gauged away and the PDOF are $(\underline{s})^{\mathrm{TT}}-2(\underline{s-1})^{\mathrm{T}}=2(s \geq 1)$, helicities $\pm s$.

For massive theories, the massless field components are augmented, for $s>2$, by traceless symmetric auxiliary fields $\left(\chi, \ldots, \chi_{\mu_{1} \cdots \mu_{s-3}}\right)$. Each divergence of the $s$-index symmetric field equations is a constraint when the remaining open indices are traceless:

\begin{tabular}{c|c} 
Fields & $(\underline{s})^{\mathrm{TT}}$ \\
+ Auxiliaries & $(\underline{0})^{\mathrm{T}}+\cdots+(\underline{s-3})^{\mathrm{T}}$ \\
\hline- Constraints & $(\underline{0})^{\mathrm{T}}+\cdots+(\underline{s-3})^{\mathrm{T}}+(\underline{s-2})^{\mathrm{T}}+(\underline{s-1})^{\mathrm{T}}$
\end{tabular}

and PDOF are $(\underline{s})^{\mathrm{TT}}-(\underline{s-2})^{\mathrm{T}}-(\underline{s-1})^{\mathrm{T}}=2 s+1$, the sum of all helicities.

For partially massless higher spin theories, there are as many possible gauge lines as divergences of the $s$-index symmetric field equations. On a line where a constraint with $t$ divergences becomes a Bianchi identity we have

\begin{tabular}{l|c}
\multicolumn{1}{c}{ Fields } & $(\underline{s})^{\mathrm{TT}}$ \\
+ Auxiliaries & $\begin{array}{c}(\underline{0})^{\mathrm{T}}+\cdots+(\underline{s-t})^{\mathrm{T}} \\
+(\underline{s-t+1})^{\mathrm{T}}+\cdots+(\underline{s-3})^{\mathrm{T}}\end{array}$ \\
\hline - Constraints & $+\frac{(\underline{s-t+1})^{\mathrm{T}}+\cdots+(\underline{s-3})^{\mathrm{T}}}{(\underline{s-2})^{\mathrm{T}}+(\underline{s-1})^{\mathrm{T}}}$ \\
- Gauge & $2(\underline{s-t})^{\mathrm{T}}$
\end{tabular}.

There are therefore $2 t+(\underline{0})^{\mathrm{T}}+\cdots+(\underline{s-2-t})^{\mathrm{T}}$ PDOF. When the new Bianchi identity is the scalar one with the maximal $t=s$ divergences, there are $2 s$ PDOF, the helicity 0 mode having been removed. For the vector Bianchi with $t=s-1$ there are $2 s-2$ PDOF and helicities $(0, \pm 1)$ are excised. For even lower values of $t$, the sum $(\underline{0})^{\mathrm{T}}+\cdots+(\underline{s-2-t})^{\mathrm{T}}$ is nonempty and corresponds to leftover auxiliary fields. The strictly massless case $t=1$ yields 2 PDOF along with decoupled but propagating auxiliary fields $(\underline{0})^{\mathrm{T}}+\cdots+(\underline{s-3})^{\mathrm{T}}$, as exemplified by the $s=3$ scalar auxiliary $\chi$.

A similar counting applies for half-integer spins: successive lower helicities are removed along gauge lines, modulo leftover auxiliaries.
Having found that all massive $s>1 / 2$ fields in (A)dS become partially massless along lines in the $\left(\mathrm{m}^{2}, \Lambda\right)$ plane, a pressing question is their precise location for $s>3$ : this determines the unitarity of the new partially massless gauge theories. For bosons, in contrast to the first order fermions, covariant derivatives in their field equations can be ordered so that the strictly massless line remains at $m^{2}=0$. This suggests that the unitary and nonunitary behaviors found in the partially massless $s=3$ and $s=$ $5 / 2$ theories, respectively, exemplify a general distinction between $s>3$ bosons and fermions. A large body of research exists for massless (A)dS higher spins (see [14], and references therein). The study of these theories in $d=5$ and their Kaluza-Klein reduction to $d=4$ ought to be relevant to the new partially massless theories described in this Letter. Whether the pattern uncovered here can be traced to a more fundamental system's expansion remains to be seen.

We thank I. Bengtsson, A. Higuchi, and R. Woodard for communications. This work was supported by NSF Grant No. PHY99-73935.

*Electronic address: deser@brandeis.edu ${ }^{\dagger}$ Electronic address: wally@brandeis.edu

[1] See, for example, S. M. Carroll, astro-ph/0004075.

[2] S. Deser and R. I. Nepomechie, Phys. Lett. 132B, 321 (1983); Ann. Phys. (N.Y.) 154, 396 (1984).

[3] K. Johnson and E. C. Sudarshan, Ann. Phys. (N.Y.) 13, 126 (1961).

[4] G. Velo and D. Zwanziger, Phys. Rev. 186, 1337 (1969).

[5] A. Higuchi, Nucl. Phys. B282, 397 (1987); B325, 745 (1989); J. Math. Phys. (N.Y.) 28, 1553 (1987).

[6] P. K. Townsend, Phys. Rev. D 15, 2802 (1977).

[7] P. A. Grassi and P. van Nieuwenhuizen, Phys. Lett. B 499, 174 (2001).

[8] S. Deser and A. Waldron, Phys. Lett. B 501, 134 (2001).

[9] K. Pilch, P. van Nieuwenhuizen, and M. F. Sohnius, Commun. Math. Phys. 98, 105 (1985).

[10] S. Deser and A. Waldron, hep-th/0103198; Phys. Lett. B 588, 347 (2001); hep-th/0105181.

[11] L. P. Singh and C. R. Hagen, Phys. Rev. D 9, 910 (1974); 9, 898 (1974); F. A. Berends, J. W. van Holten, P. van Nieuwenhuizen, and B. de Wit, Nucl. Phys. B154, 261 (1979).

[12] C. Aragone, S. Deser, and Z. Yang, Ann. Phys. (N.Y.) 179, 76 (1987).

[13] C. Fronsdal, Phys. Rev. D 18, 3624 (1978); J. Fang and C. Fronsdal, Phys. Rev. D 18, 3630 (1978); T. Curtright, Phys. Lett. 85B, 219 (1979); B. de Wit and D. Z. Freedman, Phys. Rev. D 21, 358 (1980).

[14] L. Brink, R. R. Metsaev, and M. A. Vasiliev, Nucl. Phys. B586, 183 (2000). 